\title{
Asexual reproduction in introduced and native populations of the ant Cerapachys biroi
}

\author{
DANIEL J. C. KRONAUER, ${ }^{* \dagger}+$ NAOMI E. PIERCE* and LAURENT KELLER $\ddagger$ \\ ${ }^{*}$ Museum of Comparative Zoology, Harvard University, Cambridge, MA, 02138, USA, †Laboratory of Insect Social Evolution, \\ The Rockefeller University, New York, NY, 10065, USA, \$Department of Ecology and Evolution, University of Lausanne, \\ Lausanne, 1015, Switzerland
}

\begin{abstract}
Asexual reproduction is particularly common among introduced species, probably because it helps to overcome the negative effects associated with low population densities during colonization. The ant Cerapachys biroi has been introduced to tropical and subtropical islands around the world since the beginning of the last century. In this species, workers can reproduce via thelytokous parthenogenesis. Here, we use genetic markers to reconstruct the history of anthropogenic introductions of C. biroi, and to address the prevalence of female parthenogenesis in introduced and native populations. We show that at least four genetically distinct lineages have been introduced from continental Asia and have led to the species' circumtropical establishment. Our analyses demonstrate that asexual reproduction dominates in the introduced range and is also common in the native range. Given that $C$. biroi is the only dorylomorph ant that has successfully become established outside of its native range, this unusual mode of reproduction probably facilitated the species' worldwide spread. On the other hand, the rare occurrence of haploid males and at least one clear case of sexual recombination in the introduced range show that $C$. biroi has not lost the potential for sex. Finally, we show that thelytoky in $C$. biroi probably has a genetic rather than an infectious origin, and that automixis with central fusion is the most likely underlying cytological mechanism. This is in accordance with what is known for other thelytokous eusocial Hymenoptera.
\end{abstract}

Keywords: anthropogenic introduction, asexuality, automixis, Formicidae, parthenogenesis, thelytoky

Received 2 March 2012; revision received 19 July 2012; accepted 8 August 2012

\section{Introduction}

The rise of anthropogenic environmental change and global commerce has led to the spread and establishment of an increasing number of plant and animal species worldwide. While many have no noticeable negative impact, others profoundly affect the composition of native ecosystems and are severe agricultural pests or human health hazards. In the United States alone, the annual damage caused by invasive species has been estimated at nearly $\$ 120$ billion (Pimentel et al. 2005). Although it has been a long-standing goal to

Correspondence: Daniel J. C. Kronauer; E-mail:

dkronauer@rockefeller.edu predict which species are particularly likely to become introduced and established (Kolar \& Lodge 2001; Sakai et al. 2001; Suarez et al. 2005), little overall consensus has been achieved and different factors might be important in different taxonomic groups (Kolar \& Lodge 2001; Peacock \& Worner 2008). One major initial hurdle that all colonizing species have to overcome, however, is the negative effects associated with low population densities, including inbreeding depression and the inability to find mates (Allee effects; Leung et al. 2004; Taylor \& Hastings 2005). Allee effects should be mitigated especially in organisms that have the capacity to reproduce asexually, and comparative evidence suggests that parthenogens are indeed especially common among successful invaders (Niemelä \& Mattson 
1996; Kolar \& Lodge 2001; Sakai et al. 2001; Peacock \& Worner 2008). Moreover, disturbed environments with reduced pathogen/parasite pressure might lessen selection for sex (Hoffmann et al. 2008), and the large population sizes achieved by some introduced species may reduce selection for sex further (Normark \& Johnson 2011).

Among animals, ants are among the most destructive invasive species globally, displacing native ants and profoundly affecting other organisms and the interactions between them, thereby disrupting local ecosystems (Holway et al. 2002; Lach \& Hooper-Bùi 2010). At the same time, many introduced ant species have no obvious negative ecological effects. Our current knowledge of the biology and spread of introduced ants is based on only a few well-studied species with large colony sizes, generalist diets and devastating ecological effects, that is, those that are of immediate economic concern (Suarez et al. 2010). To uncover general factors that determine the introduction potential and global introduction routes of ants, it is therefore important to take a comparative approach and study additional introduced species (Suarez et al. 2010), especially those that differ in life history from the 'classic' invasive species. Based on the information currently available, several ecological attributes are thought to correlate with an increased likelihood of successful establishment, including ground nesting, a general diet, polygynous (multiqueen) colonies and unicoloniality (Holway et al. 2002; Suarez et al. 2005, 2010; Krushelnycky et al. 2010). Over the past decade, it has also become apparent that thelytokous parthenogenesis, the production of female offspring by unmated females, is particularly common among introduced ants, while it is rare among ants in general. Thelytoky is well documented for only 13 of more than 13000 described ant species (Gotoh et al. 2012; Wenseleers \& van Oystaeyen 2011). However, at least seven of these are among the c. 200 ant species with established introduced populations: the little fire ant (Wasmannia auropunctata; Fournier et al. 2005; Foucaud et al. 2010; Rey et al. 2011), the longhorn crazy ant (Paratrechina longicornis; Pearcy et al. 2011), the yellow crazy ant (Anoplolepis gracilipes; Drescher et al. 2007), Pyramica membranifera (Ito et al. 2010), Vollenhovia emeryi (Ohkawara et al. 2006), Monomorium triviale (Gotoh et al. 2012) and Cerapachys biroi (Tsuji \& Yamauchi 1995; Ravary \& Jaisson 2004). Other parthenogenetic ants, like Mycocepurus smithii, Platythyrea punctata and Pristomyrmex punctatus, are currently not considered introduced but have unusually broad distributional ranges and are highly efficient colonizers (Rabeling et al. 2011; Seal et al. 2011).

The ant $C$. biroi has both high introductive potential and the capacity to reproduce parthenogenetically. The species belongs to the dorylomorph clade of ants, which contains close to 600 described species, including the infamous army ants (Brady 2003; Brady et al. 2006; Moreau et al. 2006). C. biroi is thought to occur natively from northern India and Nepal to southern China and Vietnam (Wetterer et al. 2012). Since the beginning of the 19th century, the species has become established on tropical and subtropical islands around the world as the only known introduced species in the entire dorylomorph clade (Wetterer et al. 2012; Fig. 1). In the

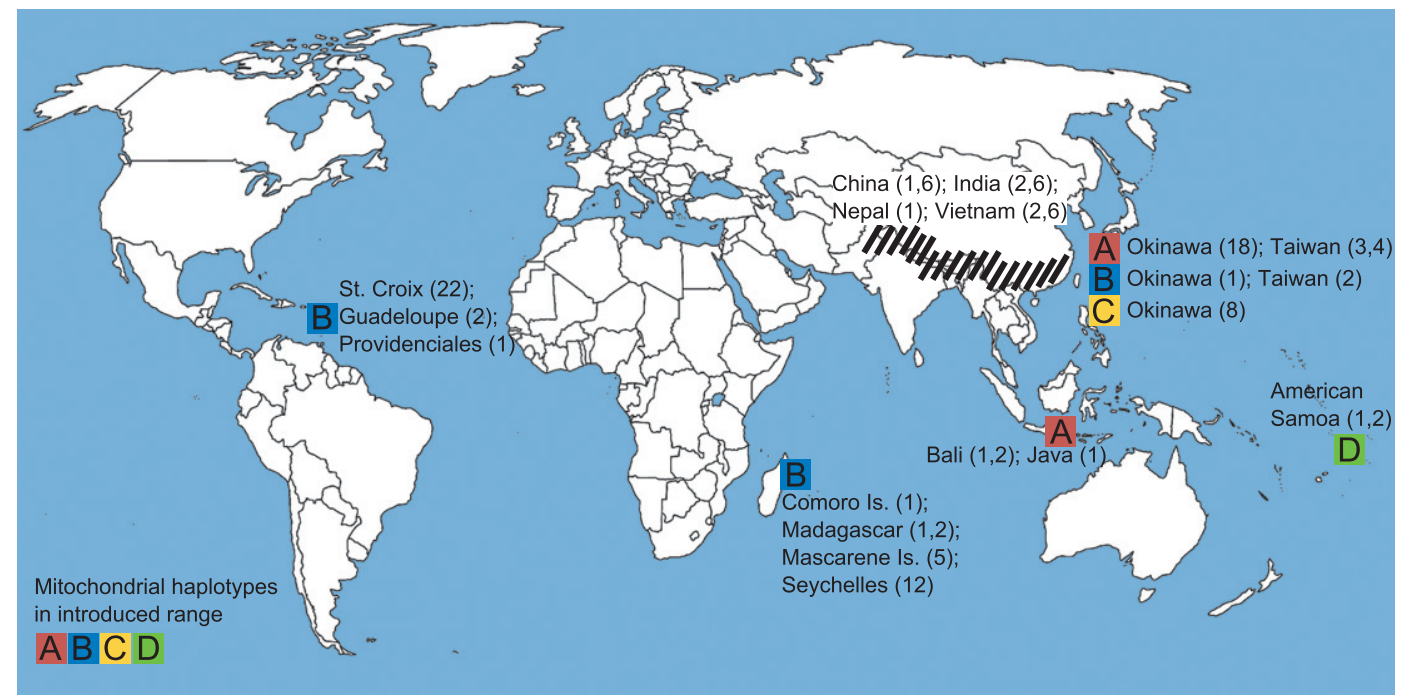

Fig. 1 Global distribution of the parthenogenetic ant Cerapachys biroi showing samples used in this study. Samples from the introduced range are colour-coded according to mitochondrial haplotype. The putative native range is hatched black. The numbers of colonies sampled are given in parentheses; the second number gives the number of individuals sampled if different from the number of colonies. 
introduced range, colonies are typically found in disturbed habitat such as parks and agricultural landscapes. Colonies are cryptic to the human observer, strictly subterranean and relatively small, usually containing only a few hundred individuals. The species has a specialized diet, raiding the nests of other ants to feed on their brood. This general biology, which is strikingly different from other well-studied introduced ants, makes C. biroi a particularly interesting study system in a comparative context. C. biroi is also the only dorylomorph known to be capable to reproduce by thelytokous parthenogenesis (Tsuji \& Yamauchi 1995; Ravary \& Jaisson 2004). Inseminated queens have never been found in this species, and when transferred to the laboratory, all workers in a colony have the capacity to lay unfertilized diploid eggs that again develop into workers (Tsuji \& Yamauchi 1995; Ravary \& Jaisson 2004). However, behavioural observations alone, especially under laboratory conditions, are insufficient to determine whether and to what extent a lineage engages in sex (Schurko et al. 2008). This problem is exacerbated in cryptic and rarely collected species like C. biroi. The role of asexual reproduction in the species' biology is therefore currently unknown.

To assess the geographical origin of introduced populations and the prevalence of parthenogenetic reproduction during the worldwide spread of C. biroi, we conducted a global phylogenetic and population genetic analysis of the species using mitochondrial and nuclear DNA sequences, as well as 30 newly developed microsatellite loci. We also transferred live field colonies to the laboratory to investigate whether workers were able to produce functional males. To assess the overall importance of asexual vs. sexual reproduction in introduced populations, we tested for several genetic correlates of asexuality (Schurko et al. 2008). We then used the microsatellite data to assign individuals to multilocus genotypes (MLGs) and to estimate the probability that recurrent MLGs (represented by individuals that are genetically identical over all assayed loci) are derived independently via sexual reproduction. MLGs were then grouped into multilocus lineages (MLLs, i.e. genotypes that are slightly different but derived from the same sexual reproduction event; Arnaud-Haond et al. 2007). The microsatellite markers were also used to distinguish between potentially functional haploid males vs. diploid males, which are sterile in most Hymenoptera (van Wilgenburg et al. 2006; Heimpel \& de Boer 2008). We tested whether parthenogenesis could be induced by symbiotic microorganisms, as is the case in many solitary Hymenoptera (Mateo Leach et al. 2009; Kageyama et al. 2012), using next generation sequencing to screen for candidate bacteria like Cardinium, Rickettsia and Wolbachia. Finally, the combined data enabled us to infer the likely cytological mechanism underlying parthenogenesis in C. biroi, in other words, to distinguish between apomixis (absence of meiosis) and different forms of automixis, during which different meiotic products fuse to restore diploidy.

\section{Materials and methods}

\section{Samples}

A total of 102 Cerapachys biroi workers from 85 colonies, and 55 males from 19 colonies, as well as one Cerapachys edentata outgroup specimen were used in this study [Table S1, Supporting Information; additional samples were used for the analysis of bacterial symbionts (see below)]. All workers were directly collected from field colonies, while males originated from laboratory colonies. We included worker samples from seven populations in the prospective native range as well as 17 introduced island populations around the world (Fig. 1, Table S1, Supporting Information). DNA quality for two specimens was low and allowed only for the sequencing of a mitochondrial DNA mini-barcode. All remaining C. biroi samples (100 workers and 55 males) were successfully genotyped at 30 novel microsatellite loci (Table S2, Supporting Information), and one specimen per colony (83 workers total) was sequenced for two mitochondrial gene fragments (1233 bp total; see below). Samples included in the final phylogenetic analysis (10 C. biroi specimens and one outgroup specimen) were also sequenced for three nuclear gene fragments (1465 bp total) in addition to mitochondrial DNA (Table S3, Supporting Information). Initial exploratory sequencing of 28 specimens from the introduced range revealed minimal sequence variation at the focal nuclear genes (Table S4, Supporting Information), and we therefore included only one representative from each of the four mitochondrial haplotypes from the introduced range in our final phylogenetic analysis.

\section{Molecular protocols}

DNA extraction. For most samples, genomic DNA was extracted from whole specimens in a final volume of $100 \mu \mathrm{L}$ using the Qiagen DNeasy Blood \& Tissue kit. Older specimens and specimens stored under poor conditions were extracted using Qiagen's QIAamp DNA Micro Kit.

DNA sequencing. We used the following primers for PCR and DNA sequencing: (i) cytochrome oxidase I (COI): LCO 1490 (Folmer et al. 1994)/HCO 2198 (Folmer et al. 1994; 50 $\mathrm{C}$ annealing temperature, 40 amplification cycles); (ii) cytochrome oxidase II (COII): AntLeu (5' AATATGGCAGATTAGTGCAATGAA 3')/Barbara (Simon et al. 1994; $48^{\circ} \mathrm{C}$ 
annealing temperature, 40 amplification cycles); (iii) wingless (Wg): wg578F (Ward \& Downie 2005)/wg1032R (Abouheif \& Wray $2002 ; 55^{\circ} \mathrm{C}$ annealing temperature, 40 amplification cycles); (iv) elongation factor $1 \alpha$ (EF1 $\alpha$ ): F2-557F (Brady et al. 2006)/F2-1118R (Brady et al. 2006; 50 $\mathrm{C}$ annealing temperature, 38 amplification cycles); (v) long wavelength rhodopsin (LR): LR143F (Ward \& Downie 2005)/ LR639ER (Ward \& Downie 2005; $50^{\circ} \mathrm{C}$ annealing temperature, 38 amplification cycles). PCR was performed in a total volume of $25 \mu \mathrm{L}$ containing 1 U QIAGEN Taq DNA Polymerase, $1 \times$ buffer, $1 \mathrm{~mm} \mathrm{MgCl}{ }_{2}, 0.25 \mathrm{~mm}$ of each dNTP, $0.8 \mu \mathrm{m}$ of each primer and $2 \mu \mathrm{L}$ DNA template.

DNA was degraded to such an extent in two samples (Cbi5 and Cbi49) that regular PCR amplification of entire fragments was impossible. For these samples, we therefore designed a primer (CbiMiniR: 5' AGAAGATTATAA TAAAAGCATGWCT $3^{\prime}$ ) to amplify a 130-bp minibarcode fragment of $\mathrm{COI}$ in combination with primer LCO 1490. PCR was performed with $45^{\circ} \mathrm{C}$ annealing temperature and 50 amplification cycles in a total volume of $25 \mu \mathrm{L}$ containing $3 \mathrm{U}$ AccuStart Taq DNA Polymerase HiFi, $1 \times$ buffer, $2.4 \mathrm{mM} \mathrm{MgSO}_{4}, 0.25 \mathrm{~mm}$ of each dNTP, $0.8 \mu \mathrm{M}$ of each primer and $10 \mu \mathrm{L}$ DNA template.

PCR products were sequenced by a commercial facility (Macrogen, South Korea). All PCR products were sequenced in both directions and sequences contained no ambiguous base calling. Sample details and GenBank accession numbers are given in Table S3 (Supporting Information).

Microsatellite genotyping. Microsatellite repeats and their flanking regions were identified from genomic reads obtained from Roche 454 shotgun sequencing libraries. PCR primers were designed using the software Primer3Plus (Untergasser et al. 2007). Microsatellite PCRs were performed in a total volume of $10 \mu \mathrm{L}$ containing $0.5 \mathrm{U}$ QIAGEN Taq DNA Polymerase, $1 \times$ buffer, $2 \mathrm{~mm} \mathrm{MgCl}_{2}$, $0.25 \mathrm{~mm}$ of each dNTP and $0.25 \mu \mathrm{m}$ of each primer. After an initial denaturation step of $3 \mathrm{~min}$ at $95^{\circ} \mathrm{C}$, samples were subject to 45 cycles of $30 \mathrm{~s}$ at $95^{\circ} \mathrm{C}, 30 \mathrm{~s}$ at $55^{\circ} \mathrm{C}$ and $30 \mathrm{~s}$ at $72^{\circ} \mathrm{C}$, followed by a final extension step of $15 \mathrm{~min}$ at $72^{\circ} \mathrm{C}$. Fluorescent-labelled PCR products were analysed on a 3730xl DNA Analyzer (Applied Biosystems), and allele calling was performed with Peak Scanner Software v1.0 (Applied Biosystems). Samples with ambiguous genotypes were genotyped repeatedly until all worker and male samples reported in this study were confidently scored for all 30 microsatellite loci. Details and GenBank accession numbers for individual microsatellite markers are given in Table S2 (Supporting Information).

\section{Analysis of DNA sequence data}

We first removed all sequences of tRNA-Leu (which directly precedes COII) from the alignment because homology could not be established unambiguously for several sites. The data set was then divided into three partitions: (i) first and second codon positions of mitochondrial DNA; (ii) third positions of mitochondrial DNA; (iii) nuclear positions. Nuclear sequences were not partitioned further given the low level of sequence divergence in the data set. Best fit models of sequence evolution were selected for each partition in the program jModelTest 0.1.1 (Guindon \& Gascuel 2003; Posada 2008) using the Akaike information criterion. Only a subset of models compatible with the program MrBayes v3.1.2 (Huelsenbeck \& Ronquist 2001; Ronquist \& Huelsenbeck 2003) was considered (by specifying three substitution schemes in jModelTest). The following models were chosen for the respective data partitions: (i) GTR + I + G; (ii) GTR + G and (iii) HKY + G. Partitioned Bayesian analysis of phylogenetic relationships was conducted in the program MrBayes. Two independent analyses were conducted to assure convergence of Markov chain Monte Carlo runs. In each case, one cold and three heated chains were run in parallel for $20 \times 10^{6}$ generations and parameters were sampled every 500 generations, resulting in a total of 40001 trees from each analysis. Average standard deviations of split frequencies were consistently $<0.01$ after $2 \times 10^{6}$ generations, indicating that independent runs had converged. The first 4000 trees from each run were therefore discarded as burn-in. Based on the remaining sample of 72002 trees from both runs combined, all potential scale reduction factors for model parameters were $\leq 1.001$, which indicates that the MCMC algorithm was effectively sampling the posterior probability distribution. We then computed a consensus phylogram with posterior probabilities based on these samples in MrBayes.

A partitioned maximum likelihood bootstrap analysis was conducted in the program GARLI 2.0 (Zwickl 2006). We set the number of generations without topology improvement required for termination to 10000 and disabled the collapsing of zero length branches. Otherwise, we used default parameter settings and conducted a bootstrap analysis with 1000 replicates. We computed a consensus phylogram with bootstrap proportions from the GARLI run in MrBayes. For the two samples for which only a mini-barcode was available, all remaining characters in the alignment were coded as missing. C. edentata (Ced1) was used as the outgroup in all phylogenetic analyses. This species is closely related to C. biroi (M. Borowiec, personal communication).

\section{Inference of asexuality}

To detect signatures of asexual reproduction, we used a multicriteria approach following Halkett et al. (2005) 
and Arnaud-Haond et al. (2007). This approach tests for the following manifestations of asexual reproduction in population genetic data: (i) occurrence of repeated MLGs; (ii) linkage disequilibrium (LD) between different nuclear markers, and between nuclear and mitochondrial markers; and (iii) deviations from Hardy -Weinberg expectations under random mating. The following analyses were performed on the worker genotypes only.

The program MLGsim2.0 (http://www.rug.nl/fmnsresearch/theobio/downloads), an updated version of MLGsim (Stenberg et al. 2003), was used to group individual microsatellite genotypes into repeatedly sampled MLGs (recurrent MLGs; i.e. genotypes that are perfectly identical over all loci). The same program was then used to test whether recurrent MLGs were a result of asexual reproduction, or whether they could be explained by insufficient discriminative power of our genetic markers. MLGsim2.0 estimates the probability $\left(P_{\text {sex }}\right)$ for each recurrent MLG that the number of observed copies would be found in a population based on the observed allele frequency and assuming random mating as the null hypothesis. MLGsim2.0 was also used to simulate 1000 populations under random mating with allele frequencies and sample sizes based on our respective data set. Our observed $P_{\text {sex }}$ values were then tested against the resulting distribution of simulated $P_{\text {sex }}$ values to determine statistical significance. We conducted the analysis on samples from the introduced range first at the global scale and then separately for each geographical region (Asia, Indian Ocean and the West Indies; the Pacific samples from American Samoa were not analysed separately given the small sample size). Despite the potential geographical substructure, these higher geographical levels of analysis were chosen based on the initial observation that recurrent MLGs occurred across different islands and even geographical regions (see below). We also conducted the analysis in MLGsim2.0 on the scale of single populations for the two islands with the largest sample sizes: Okinawa and St. Croix.

The program FSTAT 2.9.3 (Goudet 1995) was used to calculate the inbreeding coefficient $\left(F_{\text {IS }}\right.$; Weir \& Cockerham 1984) at each locus for each level of geographical sampling (see above). A significant heterozygote deficit or excess [deviations from Hardy-Weinberg Equilibrium (HWE)] was determined for each locus by testing for significant deviations of $F_{\text {IS }}$ from zero. FSTAT was also used to test for genotypic disequilibrium between all pairs of microsatellite loci within each level of geographical sampling. Both analyses were conducted with and without recurrent MLGs.

The program GenClone 2.0 (Arnaud-Haond \& Belkhir 2007) was then used to compute genetic pairwise distances between MLGs as the number of allele differences ('allele distances'). A neighbour-joining tree for all samples was computed in the program MEGA 5.03 (Tamura et al. 2011) based on the GenClone distance matrix. The same distance matrix was also used to construct a minimum spanning network for the samples from the introduced range in the program HapStar (Teacher \& Griffiths 2011). The genetic grouping of specimens based on multilocus microsatellite genotypes was then compared to the grouping based on mitochondrial haplotypes. Under strictly asexual reproduction, there should be a perfect congruence between the groupings obtained by these two sets of markers (Halkett et al. 2005).

Small differences between MLGs can arise through mutation or recombination events during parthenogenetic reproduction, rather than through sexual reproduction. It is therefore important to determine which groups of MLGs are likely descendent from the same sexual reproduction event [i.e. belong to the same asexual, or multilocus lineage (MLL)]. We grouped highly similar MLGs into MLLs following the approach outlined in Arnaud-Haond et al. (2007). Because the Okinawa population had the largest sample size among the study populations and the largest range of allelic distances between MLGs, we used this population to define a threshold value for allele distances between MLGs that were inferred to belong to the same MLL. First, we plotted pairwise microsatellite allele distances between all MLGs using the program GenClone 2.0. This revealed a strong and distinct peak of very small genetic distances (1-5 alleles across 30 diploid loci; Fig. S2, Supporting Information), indicating MLGs that are derived from the same sexual reproduction event and differ only due to mutations, partial loss of heterozygosity due to recombination during parthenogenetic reproduction or genotyping errors. We then used the $P_{\text {sex }}$ statistics on the set of identical loci between MLGs with four or five microsatellite allele differences (calculated in MLGsim2.0) to estimate the probability that these genotypes were derived from independent sexual reproduction events.

To further test for asexual reproduction within our inferred MLLs, we used FSTAT 2.9.3 to calculate $F_{\text {IS }}$ and test for significant deviations from zero for each locus within each MLL with more than two individuals (MLL1, MLL4 and MLL6). Finally, we inspected the genotypes from each MLL with more than two individuals to determine whether there were loci exhibiting a fixed heterozygous genotype. Such a pattern with some loci being fixed for heterozygous genotypes is only expected under strictly asexual reproduction within MLLs, based on a cytological mechanism that retains heterozygosity over large parts of the genome. 


\section{Bacterial diversity}

To assess whether thelytokous parthenogenesis in C. biroi could be caused by bacterial infections, we extracted genomic DNA from two pools of samples. Pools 1 and 2 each contained 15 young workers (five each from three different colonies) from asexual lineages MLL6 and MLL1, respectively. Prior to DNA extraction, samples were surface sterilized by washing in $10 \%$ bleach for c. $30 \mathrm{~s}$ and rinsed in $95 \%$ ethanol to remove residual bleach before DNA extraction. The tissue was ruptured by bead-beating for two minutes. Following lysozyme and Proteinase K digestion, DNA was extracted using a modified phenol/chloroform protocol. Genomic DNA was sent to a commercial facility (Research and Testing Laboratory, Lubbock, TX, USA) for FLX 454 pyrosequencing of a fragment that spans the variable regions V1-V3 of the bacterial $16 \mathrm{~S}$ $r R N A$ gene. Sequencing reads were initially analysed with the QIIME (Caporaso et al. 2010) core analyses script (core_qiime_analyses.py) using default parameters. After excluding all sequences with read length $<200 \mathrm{bp}$ and sequences with ambiguous base reads, 4219 reads from Pool 1 and 4593 reads from Pool 2 were included in the analysis. OTU tables were summarized at different taxonomic levels using a second QIIME script (summarize.taxa.py).

\section{Results}

\section{Geographical origin of introduced populations}

To reconstruct the geographical origin of introduced C. biroi populations, we conducted a phylogeographical analysis of samples from the native and introduced range. All samples from the introduced range were closely related and nested deeply within the samples from the putative native range. The introduced samples were most closely related to samples from Nepal and adjacent Uttarakhand in northern India, while samples from China and Vietnam were more distantly related (Fig. 2).

\section{Reproductive mode and history of introduction}

Introduced populations. Only four distinct mitochondrial haplotypes were found among samples from the introduced range (Figs 1 and 3). The high levels of pairwise sequence divergence among all mitochondrial haplotypes (1.6-3.2\%) clearly demonstrate that these represent at least four genetically distinct lineages that have been introduced from the native range, rather than differences that accumulated after introduction (assuming on the order of 100 years since first introduction and a rate of

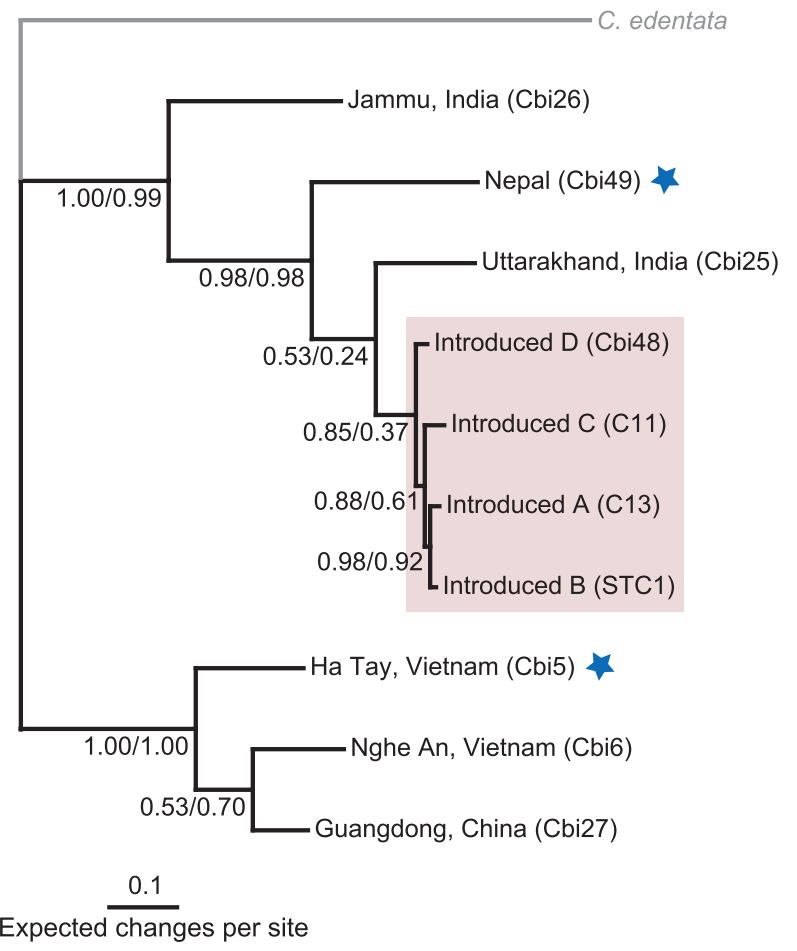

Fig. 2 Bayesian phylogram of Cerapachys biroi samples from the native and introduced range based on two mitochondrial and three nuclear gene fragments. Numbers at nodes indicate Bayesian posterior probabilities/ML bootstrap support values. The red box denotes samples from the introduced range. Samples for which only a mitochondrial mini-barcode was available are labelled with blue stars. The outgroup is depicted in grey.

sequence divergence for mitochondrial genes of c. $2 \%$ per million years).

A first inspection of the microsatellite data revealed clear deviations from the expected distribution of genotypes in panmictic populations with sexual reproduction (see Table 1 for an example of representative genotypes from Okinawa). In particular, many MLGs occurred repeatedly within populations, and at several loci, all individuals with the same mitochondrial haplotype were also heterozygous for the same pair of alleles, a pattern that can only be explained by asexual reproduction (Halkett et al. 2005; Arnaud-Haond et al. 2007).

A total of 52 MLGs (MLGs perfectly identical over all 30 loci) were detected among the 79 workers that were each collected from a different nest in the introduced range. Recurrent MLGs comprised individuals from up to eight different colonies from up to four different populations. The possibility that individuals belonging to recurrent MLGs had originated from independent sexual reproduction events was discarded in all cases, indicating that these individuals had been parthenogenetically produced (Table 2 ). 


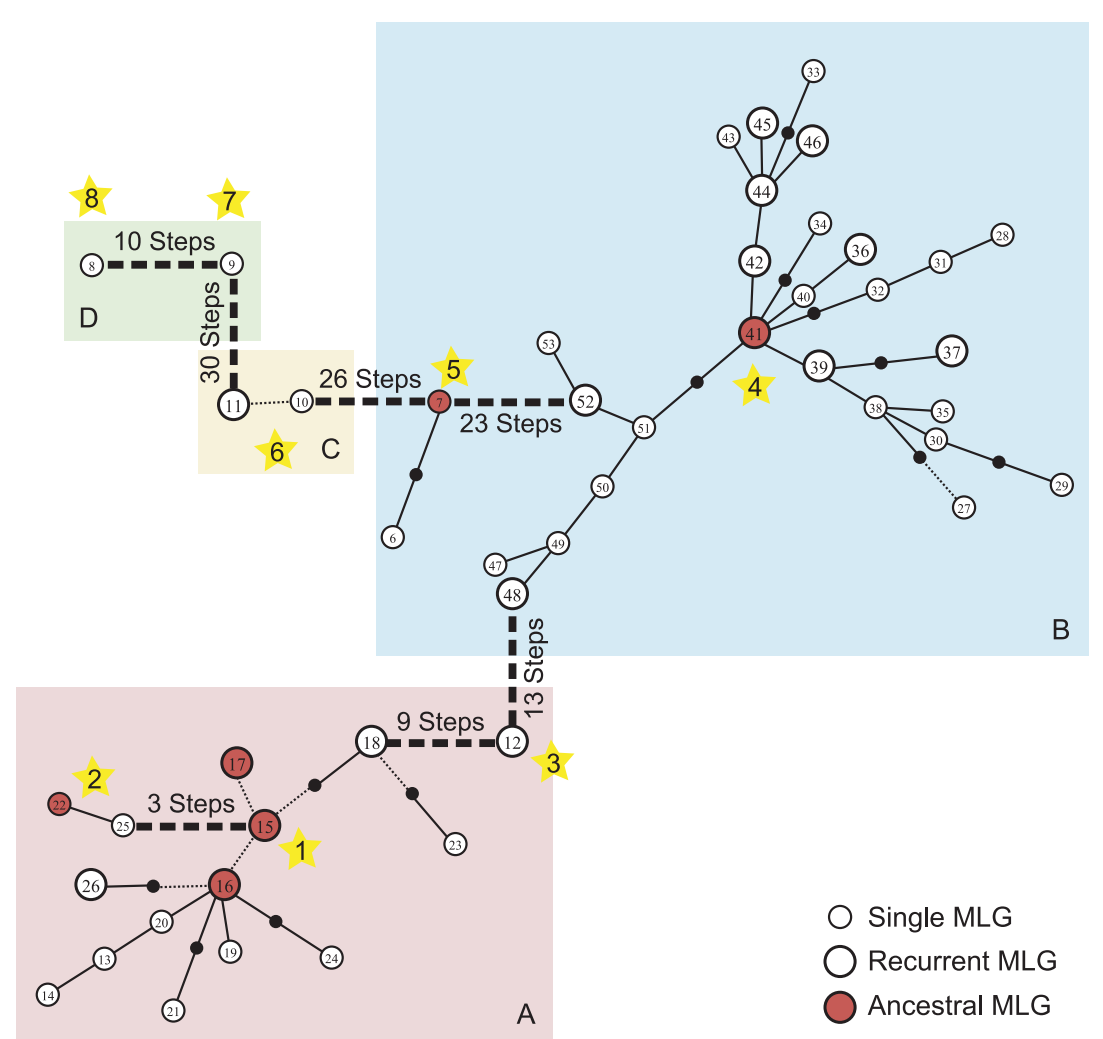

Fig. 3 Minimum spanning network of Cerapachys biroi microsatellite allelic distances from the introduced range. Each node represents one step in the network. Connections representing sexual reproduction events (three or more steps) are displayed as thick hatched lines. Connections representing size mutations of single microsatellite alleles are displayed as dotted lines, while those representing losses of heterozygosity at a single locus are displayed as solid lines. Different asexual lineages [multilocus lineages (MLLs)] are indicated by numbered stars. Ancestral multilocus genotypes (MLGs, in red) are heterozygous for all loci that show some level of heterozygosity within the respective MLL. Background shading represents the mitochondrial haplotypes of the respective samples.

The Okinawa population was used to estimate the maximum allele distance between MLGs to be considered as being asexually derived from a single original genotype (i.e. belonging to the same multilocus lineage (MLL); see above). This analysis revealed that MLGs with up to five microsatellite allele differences over the 30 loci had very low probabilities $(P<0.001)$ of being derived from independent sexual reproduction events (Arnaud-Haond et al. 2007). A difference at five microsatellite alleles was therefore used as the threshold value to group MLGs from Okinawa into the same vs. different MLLs. Further inspection of the microsatellite genotype data from the global introduced range in the light of the minimum spanning network (Fig. 3) revealed that this threshold corresponded well to subnetworks, defined as groups of MLGs in which all genotypes were connected by one or two steps. The only exception was the largest subnetwork 4, which included MLGs with up to nine allelic differences. These larger differences within this particular subnetwork were not apparent in the data from Okinawa, because only one MLG from subnetwork four was represented among the Okinawa samples. Most importantly, genetic differences among all MLGs within subnetworks could always be explained by mutations at a single allele at most, while all other genetic differences could be accounted for by losses of heterozygosity at one or more microsatellite loci, which is often a consequence of parthenogenetic reproduction (Pearcy et al. 2006; Stenberg \& Saura 2009; Kellner \& Heinze 2011; Rey et al. 2011). By contrast, subnetworks (groups of MLGs connected by more than two steps) always differed by two or more mutations or, alternatively, were separated by sexual reproduction events. We therefore defined a MLL as a group of MLGs that form a subnetwork in which all genotypes can be connected with no more than two steps.

According to this definition, the 52 MLGs clustered into eight asexual lineages (MLLs), each of which goes back to a single sexual reproduction event (Figs 3 and S1, Supporting Information). Significant heterozygote excess was detected at 11 of 12 variable loci $(92 \%)$ in MLL1 (22 individuals) and at 13 of 18 variable loci $(72 \%)$ in MLL4 (46 individuals). No significant 


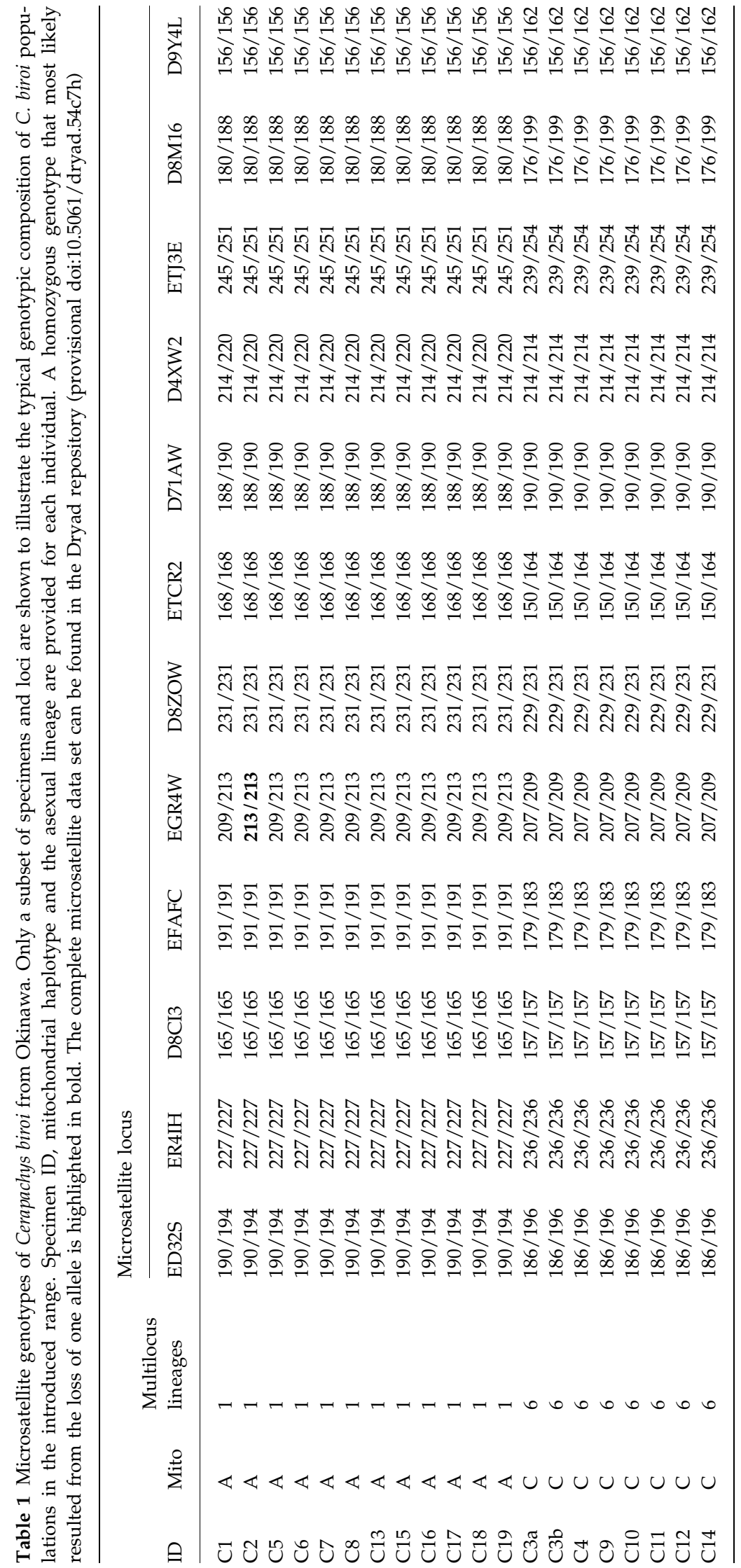


Table 2 The probability $\left(P_{\text {sex }}\right)$ that recurrent multilocus genotypes (MLGs) from the introduced range of Cerapachys biroi are derived from independent sexual reproduction events. The geographical distribution and sample size for each population are given for each MLG. $P_{\text {sex }}$ was estimated at three geographical levels ('global', 'regional' and 'local'; see main text for details). $P_{\text {sex }}$ local was only estimated for samples from Okinawa and St. Croix, the two populations with the largest sample sizes. All $P_{\text {sex }}$ values are statistically significant at $P<0.001$

\begin{tabular}{|c|c|c|c|c|}
\hline MLG & Distribution $(N)$ & $\begin{array}{l}P_{\text {sex }} \\
\text { global }\end{array}$ & $\begin{array}{l}P_{\text {sex }} \\
\text { regional }\end{array}$ & $\begin{array}{l}P_{\text {sex }} \\
\text { local }\end{array}$ \\
\hline 11 & Okinawa (7) & 0 & 0 & 0 \\
\hline 12 & Taiwan (2) & $<1 \mathrm{E}^{-14}$ & $<1 \mathrm{E}^{-14}$ & - \\
\hline 15 & Okinawa (3) & 0 & 0 & 0 \\
\hline 16 & Okinawa (5) & 0 & 0 & $<1 \mathrm{E}^{-14}$ \\
\hline 17 & Okinawa (2) & $<1 \mathrm{E}^{-14}$ & $<1 \mathrm{E}^{-14}$ & 0 \\
\hline 18 & Okinawa (2) & $<1 \mathrm{E}^{-14}$ & $<1 \mathrm{E}^{-14}$ & 0 \\
\hline 26 & Okinawa (2) & 0 & $<1 \mathrm{E}^{-14}$ & $<1 \mathrm{E}^{-14}$ \\
\hline 36 & St. Croix (5) & $<1 \mathrm{E}^{-14}$ & 0 & 0 \\
\hline 37 & Aride Is. (2) & 0 & $<1 \mathrm{E}^{-7}$ & - \\
\hline 39 & $\begin{array}{l}\text { Réunion (1), } \\
\text { Silhouette Is. (1) }\end{array}$ & $<1 \mathrm{E}^{-14}$ & $<1 \mathrm{E}^{-7}$ & - \\
\hline \multirow[t]{2}{*}{41} & $\begin{array}{l}\text { Guadeloupe (1), } \\
\text { St. Croix (3) }\end{array}$ & $<1 \mathrm{E}^{-14}$ & $<1 \mathrm{E}^{-14}$ & $<1 \mathrm{E}^{-7}$ \\
\hline & $\begin{array}{l}\text { Mauritius (2), } \\
\text { Réunion (2) }\end{array}$ & & 0 & - \\
\hline 42 & St. Croix (2) & $<1 \mathrm{E}^{-14}$ & $<1 \mathrm{E}^{-7}$ & $<1 \mathrm{E}^{-7}$ \\
\hline 44 & St. Croix (2) & $<1 \mathrm{E}^{-14}$ & $<1 \mathrm{E}^{-7}$ & $<1 \mathrm{E}^{-7}$ \\
\hline 45 & St. Croix (2) & 0 & $<1 \mathrm{E}^{-7}$ & $<1 \mathrm{E}^{-7}$ \\
\hline 46 & $\begin{array}{l}\text { Providenciales (1), } \\
\text { St. Croix (1) }\end{array}$ & $<1 \mathrm{E}^{-14}$ & $<1 \mathrm{E}^{-7}$ & - \\
\hline 48 & Silhouette Is. (2) & 0 & $<1 \mathrm{E}^{-7}$ & - \\
\hline 52 & Praslin Is. (2) & $<1 \mathrm{E}^{-14}$ & $<1 \mathrm{E}^{-7}$ & - \\
\hline
\end{tabular}

heterozygote excess was detected in MLL6, most likely reflecting the small sample size (eight individuals). A large proportion of loci were in fact fixed for a particular heterozygous allelic combination within each MLL. In MLL1, five of 12 variable loci (42\%) were fixed for a heterozygous genotype, and the same was true for seven of 18 variable loci (39\%) in MLL4 and 19 of 20 variable loci (95\%) in MLL6. This pattern can only be explained by asexual reproduction within MLLs.

In populations where a single asexual lineage dominates (West Indies, St. Croix, and Indian Ocean), the vast majority of variable loci had significant heterozygote excess (Table 3). In populations containing several asexual lineages (Global, Asia, and Okinawa), the majority of the variable loci had either significant heterozygote excess or significant heterozygote deficit (Table 3). All populations showed significant LD at most pairs of loci (Table 3). The geographical level of analysis did not affect the results qualitatively (Table 3). Microsatellite-based genetic groupings also showed perfect congruence with the four mitochondrial haplotypes

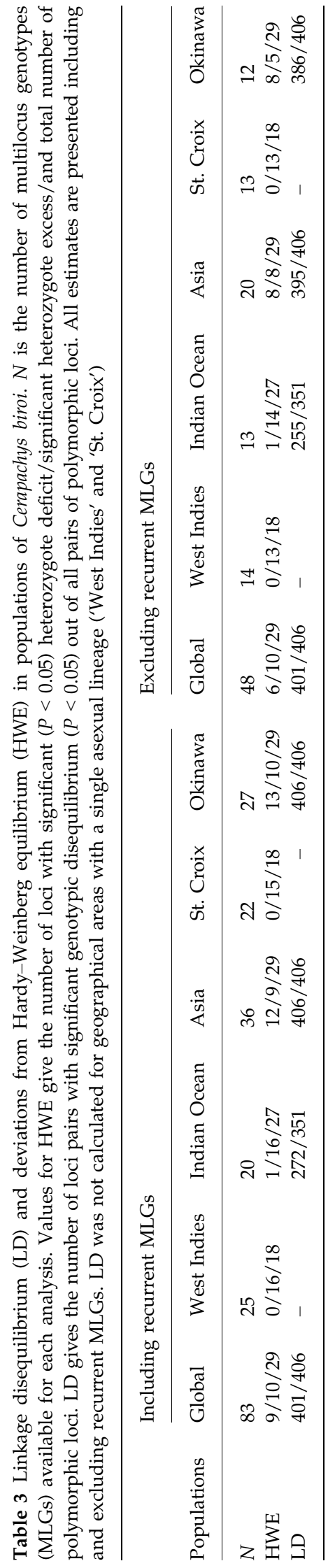


(Figs 3 and S1, Supporting Information), suggesting that both genomes are transmitted as a unit.

Three of the eight MLLs were represented by multiple colonies (MLL1, 21 colonies; MLL4, 46 colonies; and MLL6, eight colonies). Three MLLs (MLL2, MLL3 and MLL5) were only represented by a single colony each. The genotype of a second individual from each of those three colonies revealed that nestmate workers had identical or highly similar MLGs (Figs 3 and S1, Supporting Information), indicating that parthenogenetic reproduction also occurred in these nests. The single colony from American Samoa, which had a unique mitochondrial haplotype (Fig. 1), was unusual in that each of the two individuals analysed belonged to a unique MLL (MLL7 and MLL8 in Figs 3 and S1, Supporting Information). However, without additional samples from American Samoa, it is impossible to infer whether this was due to ongoing sexual reproduction or not.

A comparison among the eight MLLs from the introduced range also suggested that some lineages arose from sexual reproduction between other introduced MLLs. For example, the multilocus genotype of MLL3 was fully consistent with a single reproduction event between MLL1 and MLL4. The mitochondrial haplotype of MLL3 was also identical to that of MLL1, indicating that MLL3 almost certainly arose from an F1 female produced by sexual reproduction between an MLL1 female and an MLL4 male. Interestingly, MLL3 has only been found on Taiwan where both MLL1 and MLL4 also occur (Table S1, Supporting Information), suggesting that MLL3 arose after the introduction of $C$. biroi on this island. Similarly straightforward scenarios were not apparent for the origin of the other lineages.

The vast majority $(87 \%)$ of genetic differences between MLGs within MLLs represent transitions from heterozygosity to homozygosity at different microsatellite loci (Fig. 3). Loss of heterozygosity is often a consequence of parthenogenetic reproduction (Pearcy et al. 2006; Stenberg \& Saura 2009; Kellner \& Heinze 2011; Rey et al. 2011), and we therefore designated those MLGs that were heterozygous at all variable loci within a given MLL as ancestral (Fig. 3). In both MLL1 (MLG15, MLG16 and MLG17) and MLL4 (MLG41), the ancestral MLGs were also those that had the most replicates (Table 2). While the ancestral MLGs of MLL1 were only found on Okinawa, the ancestral MLG of MLL4 had the widest geographical distribution of any MLG, occurring on Guadeloupe, St. Croix, Mauritius and Réunion (Table 2; see also Table S1, Supporting Information). In contrast to losses of heterozygosity, size mutations (insertions/deletions) of microsatellite alleles were rare. One new size variant at a single allele across all loci arose by mutation within MLL6 and MLL4, while five new size variants arose by mutation within MLL1. Of the latter, four occurred at a single allele at a single locus, which appeared to have exceptionally high mutation rates.

Native populations. In contrast to introduced populations, colonies from the putative native range showed high diversity at mitochondrial haplotypes (six different haplotypes among six colonies and no overlap with introduced haplotypes; range of sequence divergence: $10.1-19.5 \%)$. Our sample size from native populations is limited, which precludes definitive conclusions with respect to reproductive mode. However, we were able to genotype a total of 17 individuals from four colonies for the 30 microsatellite markers (two of the six native colonies were only represented by a single poorly preserved specimen each, which did not yield sufficient DNA for microsatellite genotyping). Using the same approach as for introduced populations, this suggested that parthenogenetic reproduction is also common in the native range of C. biroi. A single MLG (MLG3 in Fig. S1, Supporting Information) was found among five workers from a colony from Vietnam, which implies strict parthenogenetic reproduction. Of the six workers from a colony from China, five belonged to a single MLG (MLG4), also implying parthenogenesis. The genotype of the sixth worker (MLG5) differed from MLG4 by one allele at each of nine loci. Given that MLG5 and MLG4 share at least one allele at each locus, it is possible that one of the two MLGs represents a case of sexual reproduction between the other MLG and a third, unknown genotype. The genotypes of two workers from a colony from Uttarakhand, India, were identical, while that of a third worker differed by a single allele (MLGs 1 and 2), clearly suggesting parthenogenesis. Finally, the three workers from a colony from Jammu, India, differed in their genotypes by two to 14 alleles (MLGs 54, 55, 56), perhaps as a result of a mix between parthenogenesis and sexual reproduction.

\section{Male production}

Live colonies from three introduced asexual lineages (MLLs 1, 4 and 6 in Figs 3 and S1, Supporting Information) were kept in the laboratory for $c .3$ years. All three asexual lineages produced adult males at very low frequencies (55 adult males collected in total: 41 from MLL1, nine from MLL4 and five from MLL6). Microsatellite genotyping revealed that 50 males (91\%) had a single allele at all thirty loci. Given the high levels of heterozygosity in diploid workers of the same asexual lineages, we conclude that these males were haploid and probably functional (van Wilgenburg et al. 2006; Heimpel \& de Boer 2008). The remaining five males (9\%; all from MLL1) were heterozygous at several loci and therefore diploid and potentially sterile. 


\section{Symbiotic microorganisms}

We obtained 4219 and 4593 bacterial reads from pools of genomic DNA extracted from MLL6 and MLL1 workers, respectively. In both cases, Actinobacteria (mainly Actinomycetales) accounted for more than half of the reads, followed by $\beta$-Proteobacteria, $\gamma$-Proteobacteria, $\alpha$-Proteobacteria and Bacilli (Fig. 4). No evidence was found for bacteria that are known to induce parthenogenesis in Hymenoptera (Cardinium, Rickettsia and Wolbachia).

\section{Discussion}

\section{History of introduction}

Cerapachys biroi has spread around the world as a consequence of human commerce at least since the beginning of the last century (reviewed in Wetterer et al. 2012). Four distinct mitochondrial haplotypes were found in our global data set from the introduced range (Fig. 1), and each was strictly associated with a group of closely related nuclear microsatellite genotypes (Fig. 3). This implies that at least four independent genetic lineages have been introduced from the native range. When compared to samples from the putative native range of the species in northern India, Nepal, Vietnam and China, it appears that the four introduced genetic lineages are all closely related phylogenetically (Fig. 2). Among the samples available for this study, they are more closely related to specimens from northern India (Uttarakhand) and Nepal than to samples from Vietnam or China (Fig. 2). However, the high levels of genetic divergence between samples from the native range raise the possibility of the existence of cryptic species. Improved sampling and a better understanding of the geographical distribution of genetic diversity are required to reliably infer the original source of the introduced populations.

Of the four introduced mitochondrial haplotypes, two were found on only a single island (haplotype $\mathrm{C}$ on

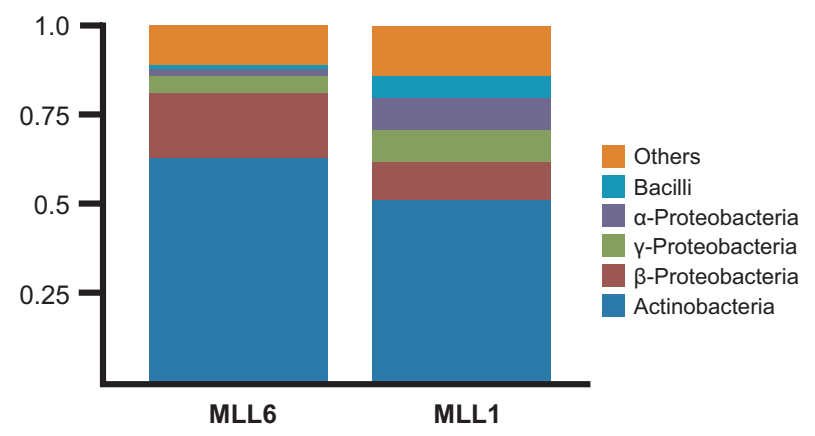

Fig. 4 Box plot of bacterial classes associated with samples from two Cerapachys biroi asexual lineages (MLL6 and MLL1).
Okinawa and haplotype D on American Samoa), while the other two (A and B) have achieved wider distribution (Fig. 1). Haplotype A was found on four different islands in Asia, and haplotype B was found around the world in the Caribbean (three islands), the Indian Ocean (nine islands) and Asia (two islands). Given the prevalence of parthenogenetic reproduction (see below) and the resulting limited genetic variation within introduced populations, it is challenging to infer the original point of introduction for the two widespread genetic lines. However, it is noteworthy that ancestral MLGs within the haplotype A clade were only found on Okinawa, suggesting Okinawa as a potential source of secondary introductions. On the other hand, the ancestral MLG within the haplotype B clade was found on different islands in the Caribbean and Indian Ocean, suggesting an original point of introduction outside of Asia. This view is based on the data presented here and might change as additional samples become available for study.

Taken together, this shows that the global spread of C. biroi has involved a combination of genetically distinct lineages being introduced from the native range, probably into different parts of the world, as well as secondary introductions within the introduced range via long-distance dispersal. Given the absence of winged queens and the strictly subterranean lifestyle, such dispersal is almost certainly human mediated in C. biroi and might occur when transported soil contains colony fragments. This overall pattern of a limited number of independent introductions followed by further expansion within the introduced range has been previously reported for other ants (Suarez et al. 2010). The fire ant Solenopsis invicta, for example, was introduced from northern Argentina into the southern US at the beginning of the last century. The global distribution of the species today can be traced back to several later introductions stemming from the original introduced range (Ascunce et al. 2011). The little fire ant, Wasmannia auropunctata, has been repeatedly introduced from its native South America to the Caribbean, from where it has then been spread globally (Foucaud et al. 2010). Finally, invasive Argentine ant (Linepithema humile) populations from different continents can be strikingly similar genetically, also suggesting a common introduction route (Brandt et al. 2009; Vogel et al. 2010). Thus, this general pattern observed in the 'classical' introduced ants also holds for C. biroi, despite the striking differences in general biology.

\section{The prevalence of asexuality}

Our genetic data show that $C$. biroi reproduces almost exclusively asexually in the introduced range. Most 
strikingly, many individuals shared identical genotypes over 30 microsatellite loci, clearly showing that those individuals are of asexual origin (Table 2). We also find pronounced deviations from HWE and strong LD at all levels of analysis (Table 3). This is expected under asexual reproduction, which results in significant deviations from HWE and in nonrandom associations between loci, creating the impression of physical linkage across the genome (Halkett et al. 2005; Arnaud-Haond et al. 2007). All MLGs from the introduced range could be grouped into only eight asexual lineages (Figs 3 and S1, Supporting Information). Within MLLs, or in populations where a single MLL dominates, we observe heterozygote excess at the majority of variable loci. This is because a large proportion of loci are either fixed for a given heterozygous genotype, or losses of heterozygosity are rare. In populations with multiple MLLs, we observe both variable loci with heterozygote excess and deficit. In this case, the loci with heterozygote deficit result from different homozygous states being fixed in the different MLLs. The perfect phylogenetic congruence between mitochondrial and nuclear DNA also implies a general lack of sexual reproduction (Figs 3 and S1, Supporting Information).

According to our genetic data, asexual reproduction is also prevalent in the native range of the species. This suggests that asexuality in C. biroi is not a consequence of anthropogenic dispersal, but a trait already present in native populations. The overall lifestyle of $C$. biroi as a specialized subterranean ant predator is rather typical for dorylomorph ants in general (Kronauer 2009) and the genus Cerapachys in particular (Hölldobler 1982). However, the species' unique biology with queenless colonies in which all individuals reproduce by thelytokous parthenogenesis, sets it apart from all other species in this clade for which life histories have been described (Tsuji \& Yamauchi 1995; Ravary \& Jaisson 2004). As a consequence, any colony fragment, when transferred to a new habitat, can potentially act as a founding propagule for a new population. This peculiar mode of reproduction has therefore most likely contributed to the emergence of C.biroi as the only introduced dorylomorph ant.

Interestingly, our results demonstrate that the potential for sexual reproduction has not been lost completely. Clear evidence for a sexual reproduction event in the introduced range was found in MLL3 from Taiwan. Furthermore, the rare occurrence of potentially functional males in laboratory reared colonies also supports the conclusion that sexual reproduction has not been lost completely in these lineages. The cooccurrence of independently derived MLGs in the colony from American Samoa and two colonies from the native range also suggests more recent sexual reproduction. However, without larger sample sizes from these populations, we cannot rule out the alternative possibility that the sampled colonies consisted of a mix of 'ancient' asexual lines. Overall, our findings highlight the fact that behavioural observations alone are not sufficient to infer absence of sexual reproduction, even in extreme cases like C. biroi, where queens have never been found.

\section{Causes and cytological mechanisms of parthenogenesis}

In Hymenoptera, thelytokous parthenogenesis can be either caused by nuclear genes or induced by cytoplasmic agents, most commonly symbiotic bacteria (Mateo Leach et al. 2009). While thelytoky induced by Cardinium, Rickettsia or Wolbachia bacteria is particularly common among solitary wasps (Mateo Leach et al. 2009; Kageyama et al. 2012), no case of microorganisminduced thelytoky is known from ants (Grasso et al. 2000; Wenseleers \& Billen 2000; Himler et al. 2009). Our screen of bacteria associated with $C$. biroi did not detect any of these candidates or any other bacteria that appeared like plausible causal agents for parthenogenetic reproduction. In fact, the majority of bacteria associated with $C$. biroi are actinomycetes, which are known as common symbionts of other ants (Ishak et al. 2011), but are not known to induce thelytoky. We therefore conclude that thelytoky in C. biroi is not caused by microorganisms and probably has a nuclear genetic basis, as is the case in honeybees, some solitary wasps and probably other ants (Lattorff et al. 2005; Mateo Leach et al. 2009; Sandrock \& Vorburger 2011).

Thelytokous parthenogenesis can have a variety of underlying cytological mechanisms (Stenberg \& Saura 2009). First, parthenogenesis can be apomictic (mitotic) or automictic (meiotic). In C. biroi, the occurrence of haploid males, which are clearly derived meiotically, indicates that meiosis is functional and therefore makes automixis more likely as the mechanism underlying thelytoky (Kellner \& Heinze 2011). In automictic parthenogenesis, the original chromosome number is restored after meiosis by one of several mechanisms, in which different meiotic products fuse. The different potential cytological mechanisms can be distinguished by their genetic consequences, that is, the rate at which heterozygosity is lost in the offspring (Stenberg \& Saura 2009). All thelytokous social Hymenoptera that have been studied in sufficient detail reproduce by automixis with central fusion (Lattorff et al. 2005; Pearcy et al. 2006; Kellner \& Heinze 2011; Rey et al. 2011). This mechanism retains heterozygosity, especially if recombination rates during meiosis are low (Lattorff et al. 2005; Pearcy et al. 2006; Stenberg \& Saura 2009; Kellner \& Heinze 2011; Rey et al. 2011). However, automixis with terminal fusion or gamete duplication are alternative 
mechanisms known from solitary Hymenoptera, and both lead to the rapid loss of heterozygosity (Mateo Leach et al. 2009). The persistence of heterozygosity in the introduced range of $C$. biroi, despite the virtual lack of sex, allows us to exclude both these latter potential mechanisms and, together with the occurrence of haploid males, suggests that thelytoky is indeed due to automixis with central fusion. However, additional work is needed to definitively distinguish between apomixis and automixis with central fusion, and to determine the precise cytological mechanism at work.

\section{Acknowledgements}

We thank the following people for generously contributing samples to this study: Himender Bharti, Marek Borowiec, Nicolas Châline, Stefan Cover, Katsuyuki Eguchi, Brian Fisher, Emmanuel Lecoutey, Bernhard Merz, Fabien Ravary, Serafino Teseo, Phil Ward, Jim Wetterer, Tony Wong and Shanyi Zhou. Marek Borowiec also confirmed species identities of samples used in this study. We are grateful to Kazuki Tsuji for hosting D.J.C.K. during his fieldwork in Okinawa and to Jim Wetterer for helpful advice in preparing D.J.C.K.'s fieldwork in St. Croix. We thank Christine La Mendola for preparing DNA samples for 454 sequencing. Jon Sanders helped invaluably with the acquisition and analysis of bacterial DNA sequence data. Comments from five anonymous reviewers and the subject editor François Rousset greatly improved the manuscript. This work was supported by a Junior Fellowship from the Harvard Society of Fellows and a Milton Fund award to D.J.C.K., NSF SES-0750480 to N.E.P., as well as a grant from the Swiss NSF to L.K.

\section{References}

Abouheif E, Wray GA (2002) Evolution of the gene network underlying wing polyphenism in ants. Science, 297, 249-252.

Arnaud-Haond S, Belkhir K (2007) GENCLONE: a computer program to analyse genotypic data, test for clonality and describe spatial clonal organization. Molecular Ecology Notes, 7, 15-17.

Arnaud-Haond S, Duarte CM, Alberto F, Serrão EA (2007) Standardizing methods to address clonality in population studies. Molecular Ecology, 16, 5115-5139.

Ascunce MS, Yang C, Oakey J et al. (2011) Global invasion history of the fire ant Solenopsis invicta. Science, 331, 1066-1068.

Brady SG (2003) Evolution of the army ant syndrome: the origin and long-term evolutionary stasis of a complex of behavioral and reproductive adaptations. Proceedings of the National Academy of Sciences of the United States of America, 100, 65756579.

Brady SG, Schultz TR, Fisher BL, Ward PS (2006) Evaluating alternative hypotheses for the early evolution and diversification of ants. Proceedings of the National Academy of Sciences of the United States of America, 103, 18172-18177.

Brandt M, van Wilgenburg E, Tsutsui ND (2009) Global-scale analyses of chemical ecology and population genetics in the invasive Argentine ant. Molecular Ecology, 18, 997-1005.
Caporaso JG, Kuczynski J, Stombaugh J et al. (2010) QIIME allows analysis of high-throughput community sequencing data. Nature Methods, 7, 335-336.

Drescher J, Blüthgen N, Feldhaar H (2007) Population structure and intraspecific aggression in the invasive ant species Anoplolepis gracilipes in Malaysian Borneo. Molecular Ecology, 16, 1453-1465.

Folmer O, Black M, Hoeh W, Lutz R, Vrijenhoek R (1994) DNA primers for amplification of mitochondrial cytochrome c oxidase subunit I from diverse metazoan invertebrates. Molecular Marine Biology and Biotechnology, 3, 294-299.

Foucaud J, Orivel J, Loiseau A et al. (2010) Worldwide invasion by the little fire ant: routes of introduction and eco-evolutionary pathways. Evolutionary Applications, 3, 363-374.

Fournier D, Estoup A, Orivel J et al. (2005) Clonal reproduction by males and females in the little fire ant. Nature, 435, 1230 1235.

Gotoh A, Billen J, Tsuji K, Sasaki T, Fuminori I (2012) Histological study of the spermatheca in three thelytokous parthenogenetic ant species, Pristomyrmex punctatus, Pyramica membranifera and Monomorium triviale (Hymenoptera: Formicidae). Acta Zoologica, 93, 200-207.

Goudet J (1995) FSTAT (Version 1.2): a computer program to calculate F-statistics. Journal of Heredity, 86, 485-486.

Grasso DA, Wenseleers T, Mori A, Le Moli F, Billen J (2000) Thelytokous worker reproduction and lack of Wolbachia infection in the harvesting ant Messor capitatus. Ethology, Ecology \& Evolution, 12, 309-314.

Guindon S, Gascuel O (2003) A simple, fast, and accurate algorithm to estimate large phylogenies by maximum likelihood. Systematic Biology, 52, 696-704.

Halkett F, Simon JC, Balloux F (2005) Tackling the population genetics of clonal and partially clonal organisms. Trends in Ecology and Evolution, 20, 194-201.

Heimpel GE, de Boer JG (2008) Sex determination in Hymenoptera. Annual Review of Entomology, 53, 209-230.

Himler AG, Caldera EJ, Baer BC, Fernandez-Marin H, Mueller UG (2009) No sex in fungus-farming ants or their crops. Proceedings of the Royal Society of London Series B, 276, 26112616.

Hoffmann AA, Reynolds KT, Nash MA, Weeks AR (2008) A high incidence of parthenogenesis in agricultural pests. Proceedings of the Royal Society of London Series B, 275, 2473 -2481 .

Hölldobler B (1982) Communication, raiding behaviour and prey storage in Cerapachys (Hymenoptera: Formicidae). Psyche, 89, 3-23.

Holway DA, Lach L, Suarez AV, Tsutsui ND, Case TJ (2002) The causes and consequences of ant invasions. Annual Review of Ecology and Systematics, 33, 181-233.

Huelsenbeck JP, Ronquist F (2001) MRBAYES: Bayesian inference of phylogeny. Bioinformatics, 17, 754-755.

Ishak HD, Miller JL, Sen R, Dowd SE, Meyer E, Mueller UG (2011) Microbiomes of ant castes implicate new microbial roles in the fungus-growing ant Trachymyrmex septentrionalis. Scientific Reports, 1, 204.

Ito F, Touyama Y, Gotoh A, Kitahiro S, Billen J (2010) Thelytokous parthenogenesis by queens in the dacetine ant Pyramica membranifera (Hymenoptera: Formicidae). Naturwissenschaften, 97, 725-728. 
Kageyama D, Narita S, Watanabe M (2012) Insect sex determination manipulated by their endosymbionts: incidences, mechanisms and implications. Insects, 3, 161-199.

Kellner K, Heinze J (2011) Mechanism of facultative parthenogenesis in the ant Platythyrea punctata. Evolutionary Ecology, 25, 77-89.

Kolar CS, Lodge DM (2001) Progress in invasion biology: predicting invaders. Trends in Ecology \& Evolution, 16, 199-204.

Kronauer DJC (2009) Recent advances in army ant biology (Hymenoptera: Formicidae). Myrmecological News, 12, 51-65.

Krushelnycky PD, Holway DA, LeBrun EG (2010) Invasion processes and causes of success. In: Ant Ecology (eds Lach L, Parr CL, Abbott KL), pp. 245-260. Oxford University Press, Oxford.

Lach L, Hooper-Bùi LM (2010) Consequences of ant invasions. In: Ant Ecology (eds Lach L, Parr CL, Abbott KL), pp. 261286. Oxford University Press, Oxford.

Lattorff HMG, Moritz RFA, Fuchs S (2005) A single locus determines thelytokous parthenogenesis of laying honeybee workers (Apis mellifera capensis). Heredity, 94, 533-537.

Leung B, Drake JA, Lodge DM (2004) Predicting invasion, propagule pressure and the gravity of Allee effects. Ecology, 85, 1651-1660.

Mateo Leach I, Pannebakker BA, Schneider MV, Driessen G, van de Zande L, Beukeboom LW (2009) Thelytoky in Hymenoptera with Venturia canescens and Leptopilina clavipes as case studies. In: Lost Sex - The Evolutionary Biology of Parthenogenesis (eds Schoen I, Martens K, van DijkP), pp. 347 -376. Springer, Heidelberg.

Moreau CS, Bell CD, Vila R, Archibald SB, Pierce NE (2006) Phylogeny of the ants: diversification in the age of angiosperms. Science, 312, 101-104.

Niemelä P, Mattson WJ (1996) Invasion of North American forests by European phytophagous insects. BioScience, 46, 741-753.

Normark BB, Johnson NA (2011) Niche explosion. Genetica, 139, 551-564.

Ohkawara K, Nakayama M, Satoh A, Trindl A, Heinze J (2006) Clonal reproduction and genetic caste differences in a queenpolymorphic ant, Vollenhovia emeryi. Biology Letters, 2, 359-363.

Peacock L, Worner SP (2008) Biological and ecological traits that assist establishment of alien invasive insects. New Zealand Plant Protection, 61, 1-7.

Pearcy M, Hardy O, Aron S (2006) Thelytokous parthenogenesis and its consequences on inbreeding in an ant. Heredity, 96, 377-382.

Pearcy M, Goodisman MAD, Keller L (2011) Sib mating without inbreeding in the longhorn crazy ant. Proceedings of the Royal Society of London Series B, 278, 2677-2681.

Pimentel D, Zuniga R, Morrison D (2005) Update on the environmental and economic costs associated with alien-invasive species in the United States. Ecological Economics, 52, 273-288.

Posada D (2008) jModelTest: phylogenetic model averaging. Molecular Biology and Evolution, 25, 1253-1256.

Rabeling C, Gonzales O, Schultz TR et al. (2011) Cryptic sexual populations account for genetic diversity and ecological success in a widely distributed, asexual fungus-growing ant. Proceedings of the National Academy of Sciences of the United States of America, 108, 12366-12371.
Ravary F, Jaisson P (2004) Absence of individual sterility in thelytokous colonies of the ant Cerapachys biroi Forel (Formicidae; Cerapachyinae). Insectes Sociaux, 51, 67-73.

Rey O, Loiseau A, Facon B et al. (2011) Meiotic recombination dramatically decreased in thelytokous queens of the little fire ant and their sexually produced workers. Molecular Biology and Evolution, 28, 2591-2601.

Ronquist F, Huelsenbeck JP (2003) MRBAYES 3: Bayesian phylogenetic inference under mixed models. Bioinformatics, 19, 1572-1574.

Sakai AK, Allendorf FW, Holt JS et al. (2001) The population biology of invasive species. Annual Review of Ecology and Systematics, 32, 305-332.

Sandrock C, Vorburger C (2011) Single-locus recessive inheritance of asexual reproduction in a parasitoid wasp. Current Biology, 21, 1-5.

Schurko AM, Neiman M, Logsdon Jr JM (2008) Signs of sex: what we know and how we know it. Trends in Ecology $\mathcal{E}$ Evolution, 24, 208-217.

Seal JN, Kellner K, Trindl A, Heinze J (2011) Phylogeography of the parthenogenetic ant Platythyrea punctata: highly successful colonization of the West Indies by a poor disperser. Journal of Biogeography, 38, 868-882.

Simon C, Frati F, Beckenbach A, Crespi B, Liu H, Flook P (1994) Evolution, weighting, and phylogenetic utility of mitochondrial gene sequences and a compilation of conserved polymerase chain reaction primers. Annals of the Entomological Society of America, 87, 651-701.

Stenberg P, Saura A (2009) Cytology of asexual animals. In: Lost Sex - The Evolutionary Biology of Parthenogenesis (eds Schoen I, Martens K, van DijkP), pp. 63-74. Springer, Heidelberg.

Stenberg P, Lundmark M, Saura A (2003) MLGsim: a program for detecting clones using a simulation approach. Molecular Ecology Notes, 3, 329-331.

Suarez AV, Holway DA, Ward PS (2005) The role of opportunity in the unintentional introduction of nonnative ants. Proceedings of the National Academy of Sciences of the United States of America, 102, 17032-17035.

Suarez AV, McGlynn TP, Tsutsui ND (2010) Biogeographic and taxonomic patterns of introduced ants. In: Ant Ecology (eds Lach L, Parr CL, Abbott KL), pp. 233-244. Oxford University Press, Oxford.

Tamura K, Peterson D, Peterson N, Stecher G, Nei M, Kumar S (2011) MEGA5: molecular evolutionary genetics analysis using maximum likelihood, evolutionary distance, and maximum parsimony methods. Molecular Biology and Evolution, 28, 2731-2739.

Taylor CM, Hastings A (2005) Allee effects in biological invasions. Ecology Letters, 8, 895-908.

Teacher AGF, Griffiths DJ (2011) HapStar: automated haplotype network layout and visualization. Molecular Ecology Resources, 11, 151-153.

Tsuji K, Yamauchi K (1995) Production of females by parthenogenesis in the ant Cerapachys biroi. Insectes Sociaux, 42, 333336.

Untergasser A, Nijveen H, Rao X, Bisseling T, Geurts R, Leunissen JAM (2007) Primer3Plus, an enhanced web interface to Primer3. Nucleic Acids Research, 35, W71-W74.

Vogel V, Pedersen JS, Giraud T, Krieger MJB, Keller L (2010) The worldwide expansion of the Argentine ant. Diversity and Distributions, 16, 170-186. 
Ward PS, Downie DA (2005) The ant subfamily Pseudomyrmecinae (Hymenoptera: Formicidae): phylogeny and evolution of big-eyed arboreal ants. Systematic Entomology, 30, 310-335.

Weir BS, Cockerham CC (1984) Estimating F-statistics for the analysis of population structure. Evolution, 38, 1358-1370.

Wenseleers T, Billen J (2000) No evidence for Wolbachiainduced parthenogenesis in the social Hymenoptera. Journal of Evolutionary Biology, 13, 277-280.

Wenseleers T, van Oystaeyen A (2011) Unusual modes of reproduction in social insects: shedding light on the evolutionary paradox of sex. BioEssays, 33, 927-937.

Wetterer JK, Kronauer DJC, Borowiec ML (2012) Worldwide spread of Cerapachys biroi (Hymenoptera: Formicidae: Cerapachyinae). Myrmecological News, 17, 1-4.

van Wilgenburg E, Driessen G, Beukeboom LW (2006) Single locus complementary sex determination in Hymenoptera: an "unintelligent" design? Frontiers in Zoology, 3, 1.

Zwickl DJ (2006) Genetic algorithm approaches for the phylogenetic analysis of large biological sequence datasets under the maximum likelihood criterion. PhD thesis, The University of Texas at Austin.

D.J.C.K. designed and performed the research, analysed the data and wrote the paper; D.J.C.K., N.E.P. and L.K. contributed new reagents/analytical tools and revised the manuscript.

\section{Data accessibility}

DNA sequences for phylogenetic analyses (accession numbers JX157193-JX157234) as well as microsatellite loci (accession numbers JX157163-JX157192) are deposited at GenBank. The final microsatellite data matrix is deposited in the Dryad repository (provisional: doi:10.5061/dryad.54c7h).

\section{Supporting information}

Additional Supporting Information may be found in the online version of this article.

Fig. S1 Neighbour-joining tree of Cerapachys biroi microsatellite allelic distances from the native and introduced range.

Fig. S2 Distribution of pairwise microsatellite allelic distances among Cerapachys biroi MLGs from Okinawa.

Table S1 Cerapachys biroi worker samples and outgroup samples used in this study.

Table S2 Microsatellite loci developed for Cerapachys biroi.

Table S3 GenBank accession numbers for DNA sequences used in the phylogenetic analyses.

Table S4 Characteristics of DNA sequences used in the phylogenetic analyses.

Please note: Wiley-Blackwell are not responsible for the content or functionality of any supporting materials supplied by the authors. Any queries (other than missing material) should be directed to the corresponding author for the article. 